\title{
Terrestrial Recovery of Extraterrestrial Materials: Providing Continued, Long-Term Sample Analysis Opportunities for Research and Mission Support
}

A White Paper submitted to the NASEM Planetary Science Decadal Survey 2023-2032 and reflecting the viewpoints of the NASA Analysis Group CAPTEM (Curation and Analysis Planning Team for Extraterrestrial Materials) and others in the sample analysis community

Hope A. Ishii ${ }^{1 *}$, Catherine M. Corrigan ${ }^{2}$, Maitrayee Bose ${ }^{3}$, Jemma Davidson ${ }^{3}$, Marc Fries $^{4}$, Juliane Gross ${ }^{5}$, James Karner ${ }^{6}$, Larry R. Nittler ${ }^{7}$, Devin L. Schrader ${ }^{3}$, Rhonda Stroud $^{8}$, Susan Taylor ${ }^{9}$ and CAPTEM.

${ }^{1}$ University of Hawaii, ${ }^{2}$ Smithsonian Institution, ${ }^{3}$ Arizona State University, ${ }^{4}$ NASA Astromaterials Acquisition and Curation Office, ${ }^{5}$ Rutgers University, ${ }^{6}$ University of Utah, ${ }^{7}$ Carnegie Institution of Washington, ${ }^{8} \mathrm{Naval}$ Research Laboratory, ${ }^{9}$ Cold Regions Research and Engineering Laboratory, retired

*Lead authors' email addresses and phone numbers:

hope.ishii@hawaii.edu; (808) 956-7755

corriganc@si.edu; (202) 633-1855

Signatories to this white paper may be found here.

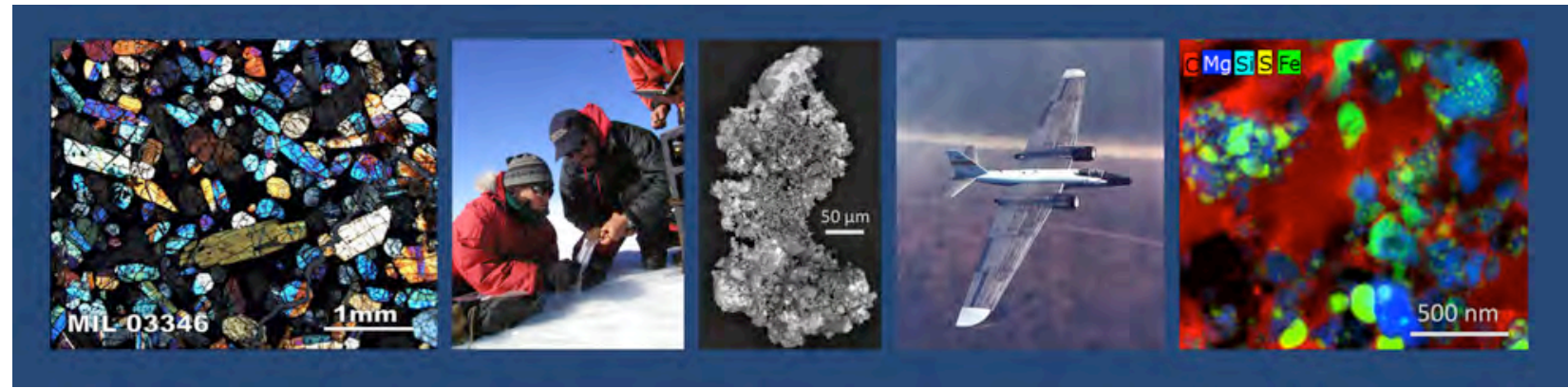




\section{EXECUTIVE SUMMARY}

This white paper summarizes the scientific importance of the terrestrial recovery and resultant collections of extraterrestrial materials. These collections provide samples from a wide range of planetary bodies, including new samples of the Moon and Mars, samples from a variety of asteroids, the only new cometary samples absent a new cometary sample return mission, and samples from bodies not accessible by sample return including those too distant to be feasible and those that have been destroyed. Extraterrestrial samples recovered on Earth have proven critical to preparation for, analyses during, and context after small body missions and planetary missions. As such, their ongoing recovery and curation are timely for maximizing science return for many missions envisioned in the coming decade, particularly those involving sample return. As a result of their serendipitous arrival on Earth, these samples can be recovered at relatively low cost, curated, and analyzed in multiple laboratories with state-ofthe-art instrumentation that is not subject to flight or quarantine constraints. We strongly advocate 1) continuing and expanding terrestrial recovery of extraterrestrial samples as an integral and critical support to small body and planetary missions and 2) developing and maintaining a high level of capability for sample curation and sample-analysis-based research by the research community. These goals require robust, sustained funding of not only recovery and curation efforts, but also of instrumentation and its support, sample-analysis-focused basic research, and fostering the next generation of scientific expertise. These goals will enable continuous mission support and rich science return throughout the coming decade and beyond.

\section{EXTRATERRESTRIAL SAMPLES ARRIVING AT EARTH ARE SERENDIPITOUS “SAMPLE RETURN"}

Collision processes on and between planetary bodies produce fragments and dust, some of which cross Earth's orbit. These fragments originate from large bodies with minimal atmospheres, including Mars and the Moon, and from small bodies, like asteroids and comets. While many fragments are altered - or completely destroyed - by melting and ablation during entry through Earth's atmosphere, sufficiently large rock fragments and sufficiently small and low-density dust particles survive with only limited alteration of their interiors. These meteorites, micrometeorites, and cosmic dust particles are invaluable samples of extraterrestrial bodies that inform our understanding of the solar system.

Extraterrestrial materials serendipitously delivered to Earth provide many of the advantages of sample return but at far lower cost. These advantages include the ability to manipulate and analyze samples using state-of-the-art instruments with high precision, accuracy, sensitivity, and resolution; to analyze very small samples with high fidelity; to demonstrate reproducibility in multiple laboratories across the globe; to reanalyze samples in the future to leverage advances in instrumentation and measurement capabilities; and finally, to apply the expertise of scientists in a range of relevant disciplines [1]. These samples do not replace mission-returned samples, for which the parent bodies are known without doubt; instead, they provide critical context (see Section 2 below).

\section{SCIENTIFIC IMPORTANCE OF TERRESTRIAL RECOVERY OF EXTRATERRESTRIAL MATERIALS}

It is difficult to overstate the significant role that terrestrially-recovered extraterrestrial materials have played, and continue to play, in developing our understanding of solar system formation and evolution, including our own planet. This is largely due to the wide variety of parent bodies from which these materials originate. Among the extraterrestrial materials in our 
cosmic dust collection are samples of undifferentiated small bodies, comets and primitive asteroids that represent the oldest building blocks of our solar system and include bits of dust inherited from the presolar cloud of dust and gas [2]. Our meteorite collections also include samples of differentiated bodies representing intermediate and advanced stages of planet formation, like Mars, the Moon, and large asteroids [3]. They include samples from beneath these parent body surfaces, not accessible by remote methods. The knowledge gained from these extraterrestrial samples delivered to Earth provides the foundation of current models for solar system formation, the basis for planetary defense against meteorite impacts, and scientific motivations for many small body and planetary missions. Ongoing terrestrial recovery of extraterrestrial materials also provides samples of bodies not in existing collections that often require revision of our understanding of the solar system and inspire new exploration.

Extraterrestrial samples recovered on Earth are critical to small body and planetary missions because they motivate and inform preparations for, analyses during, and context following missions to small bodies, moons, and planets. For the majority of missions that do not return a sample to Earth, detailed studies of meteoritic analogs on Earth provide deeper insight into mission data. A few examples, among many, have included:

a) Remote sensing of the S-asteroids Eros and Itokawa by the NEAR and Hayabusa missions, respectively, allowed the link to be made between S-type asteroids and ordinary chondrites. Analyses of the ordinary chondrites collected on Earth, using instruments like those flown on these missions, enabled comparisons to the surface measurements from these asteroids made during the missions. Final linkages were made after returned Itokawa samples were examined and compared to ordinary chondrites collected on Earth [4]. Identifying such parent bodymeteorite links effectively expands the material available for studies of that parent.

b) The M-asteroid Psyche is thought to be the stripped core of a differentiated asteroid. For the upcoming Psyche mission, researchers are comparing laboratory spectra of multiple types of iron meteorites with telescopic spectra to learn more about this asteroid before the spacecraft rendezvous. Studies show that the undifferentiated, metal-rich, CB carbonaceous chondrites are another possible match for Psyche. The hypotheses, based on studies done in Earth-based laboratories, will be tested once the spacecraft arrives at Psyche [5].

c) NASA's OSIRIS-REx mission compared the spectral properties of many types of carbonaceous chondrites with remotely sensed data of asteroid Bennu. Bennu was found to contain hydrous minerals and most closely resembles an aqueously altered CM chondrite [6]. Similarly, JAXA's Hayabusa 2 mission has determined that asteroid Ryugu bears close spectral resemblance to heated $\mathrm{Cl}$ or shocked $\mathrm{CM}$ chondrites [7]. Identifying the best meteorite match to parent asteroids enables researchers to better understand the early solar system distribution of constituent minerals and organics and constrain subsequent processing.

Terrestrially-recovered extraterrestrial materials are especially synergetic with sample return missions, both to small body and planetary targets, and contribute to maximizing science return. Earth-collected extraterrestrial materials provide scientific motivation grounded in high quality data and key comparative data collected with the same analytical instrumentation. A few illustrative examples are discussed here:

The first lunar meteorites were recognized by similarities to the samples returned by the NASA Apollo missions [8] and now provide more global information about the Moon than 
possible by missions alone. Since then, 412 lunar meteorites (ignoring pairing) have been recovered on Earth. These meteorites originate from undetermined locations on the Moon and expand our knowledge of the Moon's geologic history by allowing us to study, in great detail, samples from locations other than Apollo sites. Without these terrestrially-recovered meteorites, our understanding of the evolution and formation of the Moon would be limited to Apollo and Luna rocks, recovered from a limited geographic region on the nearside lunar surface and unrepresentative of the Moon and its geological processes. Thus, our understanding of the Earth-Moon system would be far less advanced than it is today.

The NASA Discovery mission Stardust is a comet sample return mission with science goals motivated by cosmic dust studies. Among the microscopic dust particles that settle through the Earth's atmosphere, some are strongly indicative of cometary origins. With open, porous structures and consisting of anhydrous minerals, organic matter, and amorphous silicate grains, they are consistent with water present only as ices that since sublimed away. They also contain the highest measured abundances of preserved presolar grains with isotope signatures of formation around other stars. In contrast, the comet 81P/Wild 2 samples returned by Stardust contain less presolar dust from older stars than the Earth-recovered cosmic dust that inspired the mission. They are also unexpectedly rich in rocky material that formed in hot inner solar system regions, revealing the complex transport processes operating during solar system formation [9]. Organic matter in Wild 2 samples, however, was degraded by capture. Without cosmic dust, our understanding of the diversity of materials in comets would be incomplete.

The ongoing NASA OSIRIS-REx mission will return materials from a carbonaceous asteroid. As with JAXA's Hayabusa and Hayabusa 2, these returned samples will allow for continued, indepth examination in ground-based laboratories to determine the best match for the materials in our meteoritic collections, whether the aqueously altered $\mathrm{CM}$ chondrites indicated by spectroscopy or something else entirely. It is also possible that these materials will represent an unpredicted source material, or even one that we currently do not have in our collections which, like every extraterrestrial sample newly recovered on Earth that challenges our current solar system picture, requires us to ask new questions and explore new possibilities.

Meteorite and cosmic dust collections play crucial roles in mission planning for the upcoming decade. As we prepare for new sample return missions to the Moon, Mars, asteroids, and future cometary sample returns, the samples recovered by search/recovery methods on Earth provide science questions and key insights into what to expect on these bodies. This is critical for mission preparedness and operations (including possible in situ resource utilization), including assessing which sampling sites to target to answer unresolved questions about each body. Extraterrestrial materials collections continue to be an amazing resource from which to draw these questions and inform future mission objectives.

While returned samples from Apollo enabled our recognition of lunar meteorites, future sample return missions to the Moon will, in turn, rely on those terrestrially-recovered lunar meteorites to establish optimum sampling sites. Though their source location is unknown, many of the $\sim 412$ lunar meteorites have provided new information about geologic processes on the Moon that are not recorded in the Apollo samples. New lunar sample return missions will use this expanded library of geologic compositions to select sampling sites that promote understanding of lunar geochemical evolution [10]. Currently, three lunar meteorites in the 
sample collection most likely come from the South Pole Aitken basin. These meteorites have sparked a wealth of questions and inspired proposed sample return missions to the basin. Returned samples from that site would provide ground truth to better understand, for example (a) the Cataclysm Hypothesis which, in turn, can inform us about early Solar System orbital dynamics; (b) lunar mantle compositions and heterogeneity; (c) polar volatile deposits (e.g. Artemis 2024 mission), and (d) ground truthing of orbital remote sensing.

Mars sample return mission planning will also rely on martian meteorites in terrestrial collections [11]. The primary focus for Mars sample return is on rock types formed in water-rich environments that point to past conditions on Mars that may have been conducive to life. Although no hard evidence yet exists for life on Mars, a controversy in the late 1990s about purported traces of life in the U.S. Antarctic meteorite ALH 84001 and the origin of carbonate and clay minerals in both young (nakhlite, 1.3 Ga) and old (ALH 84001, $4.1 \mathrm{Ga}$ ) martian meteorites, have driven the direction for Mars exploration for the past 15-20 years. The first recognized martian meteorite, EET A79001, was recovered as part of the fledgling U.S. Antarctic meteorite program (ANSMET) in 1979. The chemistry of gases trapped within melt pockets in the meteorite matched the composition of the Martian atmosphere measured by the Viking Landers. This is a prime example of the synergy of planetary missions with laboratory research from which we learned about both igneous processes (geochemistry and ages of events) and the processes of impact that allow samples to be launched off of bodies as large as Mars and the Moon. In the lead-up to Mars sample return, recovering and analyzing additional martian meteorites will significantly expand our geochemical understanding of the rock types present on Mars. Currently, nearly all martian meteorites are igneous rocks. The discovery of martian regolith breccia meteorites (e.g., the NWA 7034 group) provided analyses of rock types beyond the 4-5 original lithologies found in the shergottites, nakhlites, chassignites, and ALH 84001. The recovery and analysis of more regolith breccia meteorites would provide a greater understanding of martian rock types, changes in redox conditions in the martian mantle and, therefore, Mars' geologic history. This information would open the path to new questions about the red planet [12].

Terrestrial-based collections of cosmic dust provide samples of bodies that are not accessible by sample return, for example the icy Kuiper Belt bodies whose orbits are too far from Earth's orbit for sample return to be feasible. Cosmic dust will provide the only new comet samples for research until the next comet sample return, in the late 2030s or beyond. Comets formed far from the young Sun and are of enormous scientific interest as repositories of the primordial dust and ices from which the solar system formed, preserved in cryogenic conditions since they accreted. Because of their participation, together with asteroids from nearer the Sun, in bombarding the early Earth, they are also of keen interest as possible sources of volatiles and prebiotic materials, including amino acids [e.g. 13]. The Stardust mission returned samples from the coma of comet 81P/Wild 2 . The use of passive collection at $6 \mathrm{~km} / \mathrm{s}$ made it a remarkably inexpensive mission but also limited the collection of more delicate components, including those of most interest in astrobiology. Future cometary sample return missions are thus envisioned to involve direct sampling of the comet nucleus, including volatiles, and may include cryogenic sample return. Such missions would likely be New Frontiers class [14]. The next comet sample return will not occur for a decade or more. 


\section{Current Recovery efforts and challenges}

Meteorites are currently recovered in significant numbers from the few locations on Earth most amenable to their discovery and where environmental conditions limit their alteration/weathering, keeping them in a relatively pristine state. These locations include hot and cold deserts where meteorites stand out from surrounding sand, snow, or ice. Since 1976, the U.S. has been involved in funded meteorite recovery in the cold deserts of the Antarctic plateau through the Antarctic Search for Meteorites Program (ANSMET). Currently, one small team of meteorite hunters travels to the Antarctic and spends $\sim 6$ weeks camped on the ice searching for and recovering meteorites every day, weather and logistics permitting. A typical field season results in the recovery of hundreds of meteorites for the U.S. Antarctic Meteorite Collection, curated both at NASA Johnson Space Center and the Smithsonian Institution.

The meteorites recovered by ANSMET are vitally important to planetary research [15]. Their impact on planetary science (based on publications) is rivaled only by Apollo. ANSMET has supplied more known meteorites than any other source. These $23,000+$ recovered meteorites include the first recognized as lunar and martian, hundreds from 4 Vesta, and new meteorites that have challenged our understanding of the solar system. Since 1978 there have been 3600+ requests for samples and $1800+$ peer-reviewed journal articles published primarily on ANSMET meteorites; Current publication rates (up $\sim 45 \%$ since the last decadal study) predict $\sim 60$ peerreviewed publications and $\sim 200$ abstracts per year. ANSMET meteorites are in high demand because the continuous supply of new extraterrestrial materials is made available rapidly and free of charge to the world's planetary science community, unlike mission-returned samples that are not released to the research community for a period of time after return and comprise much smaller total mass. Basic research on the vast range of meteorite types returned by ANSMET has expanded our understanding of small body formation, differentiation, collisional processing, parent body alteration, and organic chemistry [16].

The primary challenge to continued ANSMET meteorite recovery efforts is the availability of stable, long-term, dedicated funding. ANSMET is currently funded by NASA, with logistical support of field activities by NSF and curation by NASA and the Smithsonian. Recovery efforts require funding of search team transport to the Antarctic, on-continent transportation, and support for the small group of researchers/geologists/mountaineers that run the program. Funding also covers search team training and the acquisition and maintenance of equipment and infrastructure for continuing recovery and curation. Therefore, ANSMET is highly cost effective, with typical recovery of $\sim 100 \mathrm{~kg}$ of samples per season, at a cost of $\sim \$ 10 / \mathrm{g}$, which is in stark contrast to the several millions of dollars per gram from sample-returned missions.

Cosmic dust derives from every dust-producing object in the Solar System, including ancient Solar System materials preserved in comets and primitive asteroids and interstellar materials [17]. Each year, the Earth sweeps up 30,000 tons of cosmic dust, but only $10 \%$ survives vaporization upon infall. Micrometeorites, $50 \mu \mathrm{m}-2 \mathrm{~mm}$ in size, comprise most of this mass and account for 50x the estimated mass of meteorites. Currently, micrometeorites are recovered from snow blowing across Antarctic ice and from sediments in the Indian Ocean. Studies of micrometeorites show that they are most similar to $\mathrm{Cl}$ and $\mathrm{CM}$ meteorites. Compositionally, $<10 \%$ are like ordinary chondrites and $<1 \%$ are achondrites. Like meteorites, some micrometeorites contain amino acids and presolar grains. Because the flux of micrometeorites has been measured, the mass of amino acids deposited to Earth and other worlds can now be 
estimated. More than 550 articles and abstracts have been published to date using materials loaned from the NASA Cosmic Dust collection.

Interplanetary dust particles, the smallest and most fragile cosmic dust particles, are 5$100 \mu \mathrm{m}$ in size [2]. This dust includes those particles most likely to originate from comets, as evidenced by the highest measured abundances of presolar grains of any meteoritic material. Interplanetary dust is recovered in the stratosphere by specialized aircraft on silicone-oil coated collector plates and curated in a dedicated cleanroom at NASA's Johnson Space Center for sample distribution to researchers worldwide. Flights can be timed to coincide with the expected settling time from known meteor showers to capture enhanced quantities of dust from specific comet and asteroid targets. For example, some dust particles collected in the stratosphere during the Earth's passage through the dust stream generated by comet 26P/Grigg-Skjellerup contained extremely high abundances of presolar material, at the percent level, attesting to the ultra-primitive nature of some cometary material [18].

Challenges for cosmic dust collection are the limited lifetime and high operating cost of the aging fleet of high-altitude aircraft, resulting in less frequent collection flights, and concerns about sample contamination from the collection medium. These challenges drive the recent development of new cosmic dust collection platforms and of new collection media. For example, silicone oil-free polymer foam collectors have been successfully flown, researchers have demonstrated low-cost high volume air samplers on the ground in remote locations in the Pacific Ocean and in Antarctica for timed collections of cosmic dust directly on dry filters, and NASA researchers are also developing a stratospheric balloon dust collection platform [19].

\section{NEED FOR STATE-OF-THE-ART CAPABILITIES FOR TERRESTRIAL RECOVERY AND ANALYSIS}

If stored under non-ideal conditions, extraterrestrial samples degrade over time, causing uncertainty in provenance and evolution of some features, especially organics, volatiles and hydrated materials that are of particular interest to several missions planned and proposed in the coming decade. To mitigate these issues, extraterrestrial material recovery efforts should be continued and be expanded to take advantage of improvements in sample detection, collection, handling, storage, and curation practices. In the past decade, new methods of minimizing terrestrial contamination and preserving meteoritic samples in a pristine state have been developed. These include cold curation, gas cabinets, and improved monitoring for organic contamination in curation facilities. In addition, new detection networks like the Global Fireball Observatory, an international collaboration using networked cameras, have been employed to detect meteorite falls for rapid recovery of samples with less terrestrial weathering and to trace the meteorite path back to its likely origin.

Because of the specialized requirements to avoid contamination and preserve extraterrestrial samples in as pristine a state as possible, maintaining sample curation capabilities, including sample handling, classification, and proper curation expertise and facilities is essential $[19,20]$. These facilities must be maintained separately from returned sample facilities in order to avoid cross-contamination. Current sample curation capabilities are distributed among multiple types of institutions, government laboratories and museums, academic institutions, research institutions, and private museums. These capabilities are not entirely interchangeable since various facilities specialize in handling specific sample types. For example, the Smithsonian processes all iron meteorites returned by ANSMET and provides 
long-term curation of all ANSMET samples, and NASA's Astromaterials Curation operates a specialized clean room laboratory for handling and curating cosmic dust. A number of academic institutions, private museums, and research institutions, in the US and abroad, also curate collections of extraterrestrial materials.

Similarly, state-of-the-art analytical capabilities, including instrumentation and expertise, are necessary to determine the properties and characteristics of extraterrestrial materials. As sample return missions bring back small masses of materials, instrumentation must be capable of high precision, accuracy, sensitivity, and resolution $[1,19,20]$. Analyses cover the gamut from highly sensitive chemical analyses, to high resolution mineralogic and petrographic imaging and mapping, to spectroscopic studies over a range of wavelengths and length scales. Current analytical capabilities are even more widely distributed across institutions than curation capabilities due to the breadth of the research enterprise, allowing for the application of highly specialized analytical techniques and the expertise of scientists across a range of diverse disciplines, including meteoritics, analytical chemistry, biology, and physics. Engaging students in research on terrestrially-recovered extraterrestrial materials using state-of-the-art tools is the primary means of motivating and educating the next generation of planetary scientists.

\section{CONCLUSIONS/RECOMMENDATIONS}

In order to prepare for, analyze data during, and provide context after small body and planetary missions, including, but not limited to, sample return missions, we strongly advocate 1) continuing and expanding terrestrial recovery of extraterrestrial samples, including dedicated funding for Antarctic sample recovery and funding for new modes and methods of cosmic dust collection, and 2) maintaining a high level of capability for sample curation and sample-analysisbased research throughout the community. This requires robust funding of recovery and curation efforts, instrumentation, sample-analysis-focused basic research, and scientific expertise, including training future scientists. The benefits will accrue in more comprehensive mission support and enriched science return throughout the coming decade and far beyond.

\section{REFERENCES}

1. Stroud R. et al. (2020) Planetary Decadal Survey white paper. 2. Bradley J. P. (2014) "Interplanetary Dust Particles" in Treatise on Geochemistry, $2^{\text {nd }}$ ed, v1. 3. Righter K. et al., eds. (2014) 35 Seasons of Antarctic Meteorites (1976-2010). 4. Nakamura T. et al. (2011) Science 333:1113-13116. 5. Elkins-Tanton L. T. et al. (2020) JGR Planets 125, e2019JE006296. 6. Hamilton V. E. et al. (2019) Nature Astron 3:332-340. 7. Kitazato K. et al. (2019) Science 364:272-275. 8. Antarctic Meteorite Newsletter 6 (1), Feb. 1983. 9. Brownlee D. E. (2014) AnnRevEPS 42:179-205. 10. Tartèse R. et al. (2019) Space Sci Rev 215:54. 11. McSween H. Y. et al. (2020) Planetary Decadal Survey white paper. 12. Udry A. et al. (2020) Planetary Decadal Survey white paper. 13. Callahan M. P. (2011) PNAS 108: 13995-13998. 14. Westphal A. (2020) Planetary Decadal Survey white paper. 15. Wadhwa M., McCoy T. J., and Schrader D. L. (2020) AnnRevEPS 48:10.1-10.26. 16. Bose M. and Jacobsen S. (2020) Planetary Decadal Survey white paper. 17. Taylor S. et al., eds. (2016) Elements 12 (3) and references therein. 18. Busemann H. et al. (2009) EPSL 288:44-57. 19. McCubbin F. M. et al. (2020) Planetary Decadal Survey white paper. 20. NASEM (2019) Strategic Investments in Instrumentation and Facilities for Extraterrestrial Sample Curation and Analysis. Washington, DC: National Academies Press. 